\title{
3D-Printable Materials for Microbial Liquid Culture
}

\author{
Matthew E. Walsh, Alla Ostrinskaya,, Morgan T. Sorensen,2, David S. Kong,, and Peter A. Carr ${ }^{1}$
}

\begin{abstract}
Additive manufacturing is providing the opportunity to easily fabricate complex structures for biological applications, such as growing microbial cultures. To best determine 3D-printable materials suitable for liquid microbial growth, culture tubes were printed that mimic the shape and dimensions of a standard commercial tube. Ten different printed materials were assessed in conjunction with multiple 3D printing platforms, and their performance to standard commercial polystyrene culture tubes was compared. In each test, a K-12 strain of the common laboratory bacterium Escherichia coli was monitored for growth over $24 \mathrm{~h}$ under normal conditions of temperature and agitation. Growth inhibition was observed for some printed materials, and mass spectrometry was used to identify two leached chemicals that inhibited bacterial growth. Based on this assessment, three of the materials are recommended for consideration in prototyping 3D-printed culture environments.
\end{abstract}

\section{Introduction}

THE RECENT INCREASED ACCESSIBILITY OF 3D printing has given rise to many creative advancements in both printable materials and applications. ${ }^{1}$ Materials suitable for $3 \mathrm{D}$ printing include plastics, ${ }^{2,3}$ metals, ${ }^{4}$ and even glass. ${ }^{5,6}$ Recently, the biomedical field has seen great benefit from the increased proliferation of 3D printing. 3D-printable biomedical devices can be fabricated with customized geometries, creating a path to personalized devices specific to an individual's morphology or physiology. ${ }^{7}$ In addition to clinical applications, 3D printing technology can be used to fabricate devices at the milli- and microfluidic "lab-on-a-chip" scale to investigate biological phenomena at the cellular or molecular level. ${ }^{8}$ However, the impact of many of the novel materials used in 3D printing on biomolecules or cells has not been thoroughly characterized. Zhu et al. has noted that certain 3D printing materials alter the morphology of zebra fish embryos, ${ }^{8}$ while Lücking et al. performed mammalian cell culture in 3Dprinted growth plates. ${ }^{9}$ With the potential of utilizing 3D printing for biological applications, including not only medical devices but also diagnostic platforms and benchtop laboratory experiments, there is a growing need to understand how printable materials interact with cells and organisms of interest. 3D printing is a cost-effective way to rapidly prototype devices in small batches in an on-demand manner. To our knowledge, there is no published literature, in which the authors culture microbes in 3D-printed devices or vessels, warranting investigation into the suitability of materials for such applications.

\section{Materials and Methods}

\section{Growth conditions}

An overnight culture of Escherichia coli strain JW0740-3 (supplied by The Coli Genetic Stock Center, Yale University, New Haven, CT) was inoculated with a single colony in $5 \mathrm{~mL}$ of sterile LB Broth $+50 \mathrm{mg} / \mathrm{mL}$ kanamycin and shaken at $37^{\circ} \mathrm{C}$. Before use, each printed tube was UV-irradiated for $15 \mathrm{~min}$. After irradiation, $5 \mathrm{~mL}$ of sterile LB Broth $+50 \mathrm{mg} /$ $\mathrm{mL}$ kanamycin were inoculated with $100 \mu \mathrm{L}$ of the overnight culture and considered time 0 . Tubes were incubated in a $37^{\circ} \mathrm{C}$ shaking incubator between $\mathrm{OD}_{600}$ measurements.

\section{Optical density measurements}

An Eppendorf BioPhotometer (Eppendorf, Hamburg, Germany) was used with $8.5 \mathrm{~mm}$ center height UVettes (Eppendorf, Cat. no.: 952010069) with a $100 \mu \mathrm{L}$ sample volume. Measurements were made at $0,2,4,6,8$, and $24 \mathrm{~h}$. For Figure 2, and with the exception of the "Tango Plus" tubes,

\footnotetext{
${ }^{1}$ Massachusetts Institute of Technology Lincoln Laboratory, Lexington, Massachusetts.

${ }^{2}$ Department of Chemistry, Augustana University, Sioux Falls, South Dakota.

(c) Matthew E. Walsh et al., 2016; Published by Mary Ann Liebert, Inc. This Open Access article is distributed under the terms of the Creative Commons Attribution Noncommercial License (http://creativecommons.org/licenses/by-nc/4.0/) which permits any noncommercial use, distribution, and reproduction in any medium, provided the original author(s) and the source are credited.
} 
samples were diluted 1:5 in media at 6,8 , and $24 \mathrm{~h}$. All samples were blanked against media incubated in a tube of the given material. For Figure 4, samples were diluted if they were above $\mathrm{OD}_{600}=1.000$ and blanked against unused media.

\section{Mass spectrometry}

Reagents. Ethyl-methacrylate, phosphate-buffered saline, amino acids standard, and formic acid were purchased from Sigma-Aldrich (St. Louis, MO). Diethyl-dimethylaminomethylene malonate was purchased from Alfa-Aesar (Ward Hill, MA). Solvents included LC/MS grade methanol and water and were purchased from VWR (Radnor, PA).

Instruments. To identify residual chemicals from the FormLabs "Flexible" test tubes, we used an Agilent 6520 quadrupole time-of-flight mass spectrometer (Agilent Technologies, Santa Clara, CA) equipped with an electrospray ionization (ESI) source and Agilent MassHunter ${ }^{\circledR}$ workstation software version B.0.5.00. Samples were diluted 10-fold in methanol and introduced into the mass spectrometer by direct syringe infusion at the rate of $10 \mu \mathrm{L} / \mathrm{min}$. The mass spectrometer parameters used for data acquisition were: source gas temperature: $200^{\circ} \mathrm{C}$, drying gas flow: $5 \mathrm{~L} / \mathrm{min}$, nebulizer pressure: $15 \mathrm{psi}$, fragmentor voltage: $100 \mathrm{~V}$, and the inlet capillary voltage: $4000 \mathrm{~V}$. All spectra were acquired in positive polarity.

To determine the amount of amino acids in the media, we used ABSCIEX 4000 QTRAP hybrid triple quadrupole linear ion trap mass spectrometer equipped with an ESI probe and combined with 1100/1200 Agilent Capillary LC system. The following mass spectrometer parameters were used: source temperature: $500^{\circ} \mathrm{C}$, an ion spray voltage: $5500 \mathrm{~V}$, curtain gas flow: $10 \mathrm{psi}$, source gas flow: $40 \mathrm{psi}$, entrance potential: $10 \mathrm{~V}$, and collision cell exit potential: $15 \mathrm{~V}$. The data were acquired in positive polarity in multiple reactions monitoring mode. All mass-to-charge ratio (Q1 mass, Q3 mass) used for amino acid detection are listed in Table 1 , as well as declustering potential and collision energy, which were optimized for each amino acid individually.

TABle 1. ABSCIEX 4000 Parameters FOR AMINo ACID ANALYSIS

\begin{tabular}{lcccc}
\hline Analyte & $Q 1(\mathrm{~m} / \mathrm{z})$ & $Q 3(\mathrm{~m} / \mathrm{z})$ & $D P$ & $C E$ \\
\hline Glycine & 76 & 30 & 50 & 10 \\
Alanine & 90 & 44 & 50 & 17 \\
Valine & 118 & 72 & 50 & 16 \\
Serine & 106 & 60 & 50 & 16 \\
Proline & 116 & 70 & 50 & 25 \\
Threonine & 120 & 74 & 50 & 16 \\
Aspartic acid & 134 & 88 & 50 & 15 \\
Glutamic acid & 148 & 84 & 50 & 23 \\
Leucine/Isoleucine & 132 & 86 & 50 & 20 \\
Lysine & 147 & 84 & 50 & 25 \\
Methionine & 150 & 104 & 50 & 15 \\
Phenylalanine & 166 & 120 & 50 & 18 \\
Arginine & 175 & 70 & 50 & 32 \\
Histidine & 156 & 110 & 50 & 20 \\
Tyrosine & 182 & 136 & 50 & 18 \\
Cystine & 241 & 120 & 50 & 26 \\
\hline
\end{tabular}

$\mathrm{CE}$, collision energy; DP, declustering potential.
Samples were infused directly in to an ESI probe. The quantitation of amino acids in the media was done with an external standard calibration curve (Cat. no.: AAS18-5ML; Sigma-Aldrich), where concentration of amino acid standards varied from 0.004 to $0.4 \mathrm{ng} / \mathrm{mL}$. All standards and samples were diluted with methanol.

\section{Results}

We 3D printed standard $14 \mathrm{~mL}$ culture tubes in a variety of materials and investigated the suitability of these materials for bacterial growth using a conventional K-12 strain of E. coli, a model organism widely used for microbial research. $E$. coli was chosen for this work as it is one of the most commonly used laboratory microbes, known for robust growth under a variety of conditions. Our rationale was that if a particular material is incompatible with growing this organism, it would likely be unsuitable for many other microbes as well. Culture tubes were designed in SolidWorks (Fig. 1), modeled from $14 \mathrm{~mL}$ polystyrene round bottom $17 \times 100 \mathrm{~mm}$ culture tubes with a $1 \mathrm{~mm}$ wall thickness. The .stl files for tube and cap are included in the supplementary material (Supplementary Data are available online at www.liebertpub.com/3dp). Using this design, we fabricated tubes from 10 different materials. Properties of these printed tubes are given in Table 2 .

Tubes printed from four of the materials were purchased through the service provided by Shapeways (www.shapeways .com) through their online ordering process. Thus, for these materials, the user need not perform his or her own 3D printing. These printed tube materials were: "Elasto Plastic" (Shapeways' flexible material, printed using an SLS method), "Extreme Detail"' (highest print step resolution, $16 \mu \mathrm{m}$ ), "Frosted Acrylic,", and "White Strong and Flexible." Initial examination of these tubes revealed that tubes printed with "Elasto Plastic" allowed rapid evaporation of media (consistent with Shapeways' recommendation that this material should not be used for liquids). The "Extreme Detail"' tubes exuded a sticky yellow residue after storage at room temperature for 3 months. The "White Strong and Flexible" tubes also allowed evaporation of liquid and in addition shed fine white particles into the media. For the above reasons, these three types of tubes were judged not suitable for experiments involving bacterial growth in liquid media.
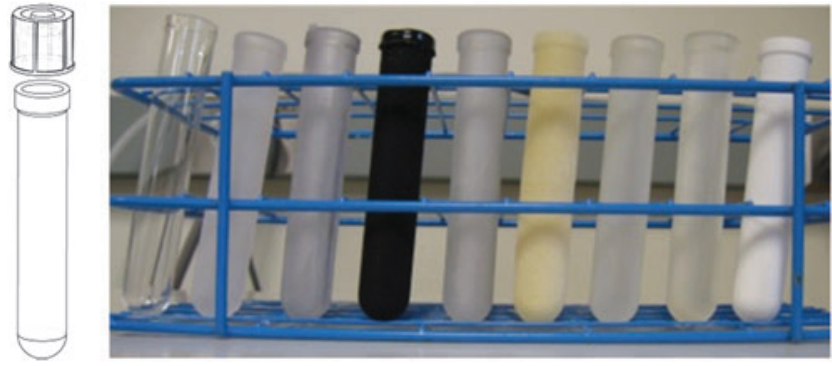

FIG. 1. 3D-printed test tubes. Left: SolidWorks schematic of tube design with cap. Right: Test tubes printed of various materials: (from left to right) VWR Polystyrene, FormLabs "ClearV2," FormLabs "Flexible," "TangoBlack," "VeroClear," Shapeways "Elasto Plastic," Shapeways "Extreme Detail," Shapeways "Frosted Acrylic," and Shapeways "White Strong and Flexible." 
Table 2. Properties of 3D-Printable Materials Evaluated for Microbial Liquid Culture

\begin{tabular}{|c|c|c|c|c|c|}
\hline Material & Flexible & Transparency & $\begin{array}{l}\text { Z-axis print } \\
\text { resolution }(\mu m)\end{array}$ & $\begin{array}{c}\text { Growth } \\
\text { comparable } \\
\text { to control }\end{array}$ & $\begin{array}{c}\text { Liquid } \\
\text { compatible }\end{array}$ \\
\hline Polystyrene Control & No & Transparent & - & Yes & Yes \\
\hline FormLabs Clear V2 & No & Translucent & 25 & No & Yes \\
\hline FormLabs Flexible & Yes & No & 100 & No & Yes \\
\hline Stratasys MED610 ${ }^{a}$ & No & Translucent & 16 & Yes & Yes \\
\hline Stratasys TangoBlack & Yes & No & 16 & - & Yes \\
\hline Stratasys TangoPlus & Yes & Translucent & 16 & No & Yes \\
\hline Stratasys VeroClear ${ }^{a}$ & No & Translucent & 16 & Yes & Yes \\
\hline Shapeways Elasto Plastic & Yes & No & 150 & - & No \\
\hline Shapeways Extreme Detail & No & Translucent & 16 & - & Yes \\
\hline Shapeways Frosted Acrylic ${ }^{a}$ & No & Translucent & 20 & Yes & Yes \\
\hline $\begin{array}{l}\text { Shapeways White Strong } \\
\text { and Flexible }\end{array}$ & No & No & 120 & - & No \\
\hline
\end{tabular}

Entries in bold remained both physically intact and supported microbial growth at a rate similar to that of the control.

A FormLabs (Somerville, MA) Form 1+ printer was used to print tubes in "Flexible" and "ClearV2" resins. This printer has a cost of $\sim \$ 3000$, making it accessible for many initial laboratory investigations. The third and final means of production were the Stratasys (Billerica, MA) Connex 350, which costs $\sim \$ 250,000$ and was used to print "Tango Black," "Tango Plus," "Med610," and "VeroClear" resins. The Connex 350 build platform is more than four times the size of the Form 1+ build platform, and thus, the Connex 350 can print more tubes per unit time than the FormLabs printer, but is considerably more expensive to purchase and maintain.

On the Connex 350, tubes were typically printed parallel to the print surface, which resulted in support material on the inside of the tube that was cleared using a power washer. The "TangoBlack" material required printing in a perpendicular orientation to the build platform to avoid support material deposition inside the tube, as the integrity of the tube did not hold up to the power washing otherwise required to remove the support material. However, the perpendicularly printed tubes tore after just a few hours during incubation in a $37^{\circ} \mathrm{C}$ shaking incubator and, thus, were not used in growth experiments. According to the Stratasys PolyJet ${ }^{\mathrm{TM}}$ Materials Data Sheet, the "TangoPlus" resin has an elongation at break (170-220\%) which is roughly $4 \times$ higher compared with the simple "Tango" material (45-55\%). The "TangoPlus" tubes maintained structural integrity throughout the $24 \mathrm{~h}$ shaking and incubation. The FormLabs "Flexible" material and the Shapeways "Elasto Plastic" material are both flexible in that they undergo elastic deformation when squeezed; however, they lack the elastic elongation properties of the Tango family of materials. We also note that the "MED610" resin is the only resin tested to be marketed as "biocompatible" in accordance with standard DIN EN ISO 10993-1:2009.

Once 3D printed, the tubes were used to culture E. coli strain $\Delta$ galK $\left(\right.$ JW0740-3) ${ }^{10}$ in kanamycin $(50 \mathrm{mg} / \mathrm{mL})$ supplemented LB Broth (Lennox Formulation, Cat. no.: L3022; Sigma-Aldrich) over the course of $24 \mathrm{~h}$ with shaking at $37^{\circ} \mathrm{C}$. The caps used for all tubes were the same polystyrene caps supplied with the control tubes (refer to Materials and Methods for detailed measurement protocol). Figure 2 shows the endpoint $\mathrm{OD}_{600}$ measurement of culture grown in six types of 3D-printed tubes and control polystyrene tubes, as well as starting $\mathrm{OD}_{600}$ measurements at $2,4,6$, and $8 \mathrm{~h}$. For each material, three tubes were printed and duplicate $\mathrm{OD}_{600}$ measurements from each tube were taken at the respective time points.

The various materials can be grouped into two categories based on growth pattern. The first group are those materials that have similar growth patterns over the first $8 \mathrm{~h}$ to that of

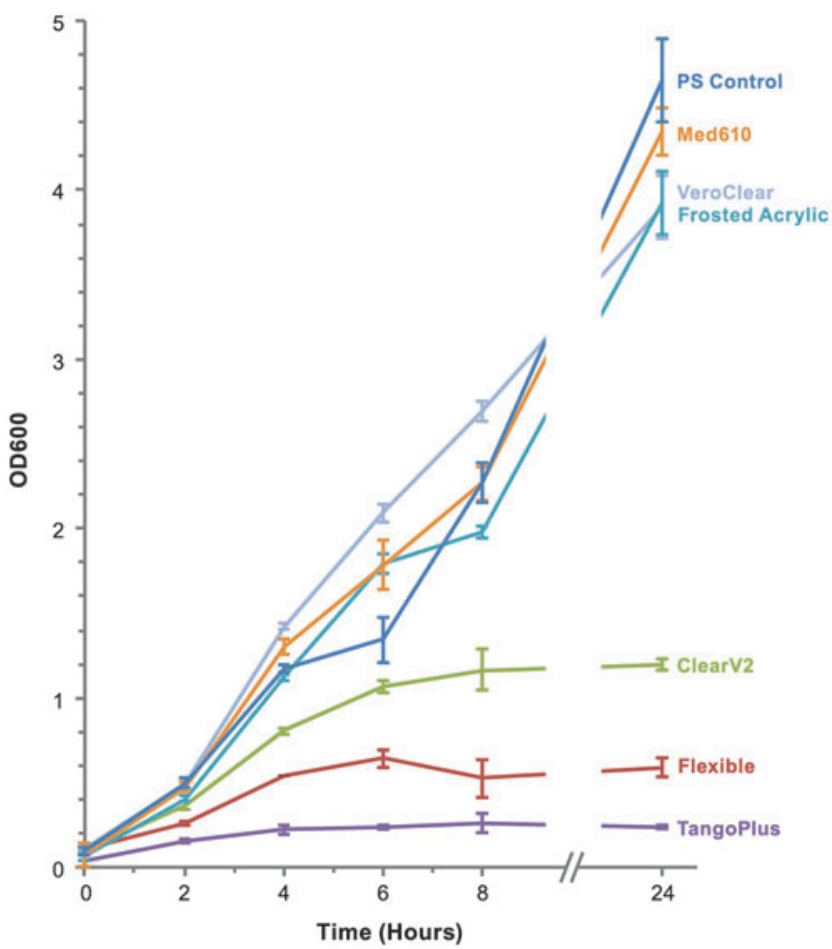

FIG. 2. Growth curve of KEIO collection strain JW0740-3 in polystyrene control, "MED610," "VeroClear," "Frosted Acrylic," "ClearV2," "Flexible," and "TangoPlus" tubes over the course of $24 \mathrm{~h}$. Error bars represent $\pm 1 \mathrm{SD}$ of three replicate cultures grown simultaneously. SD, standard deviation. 

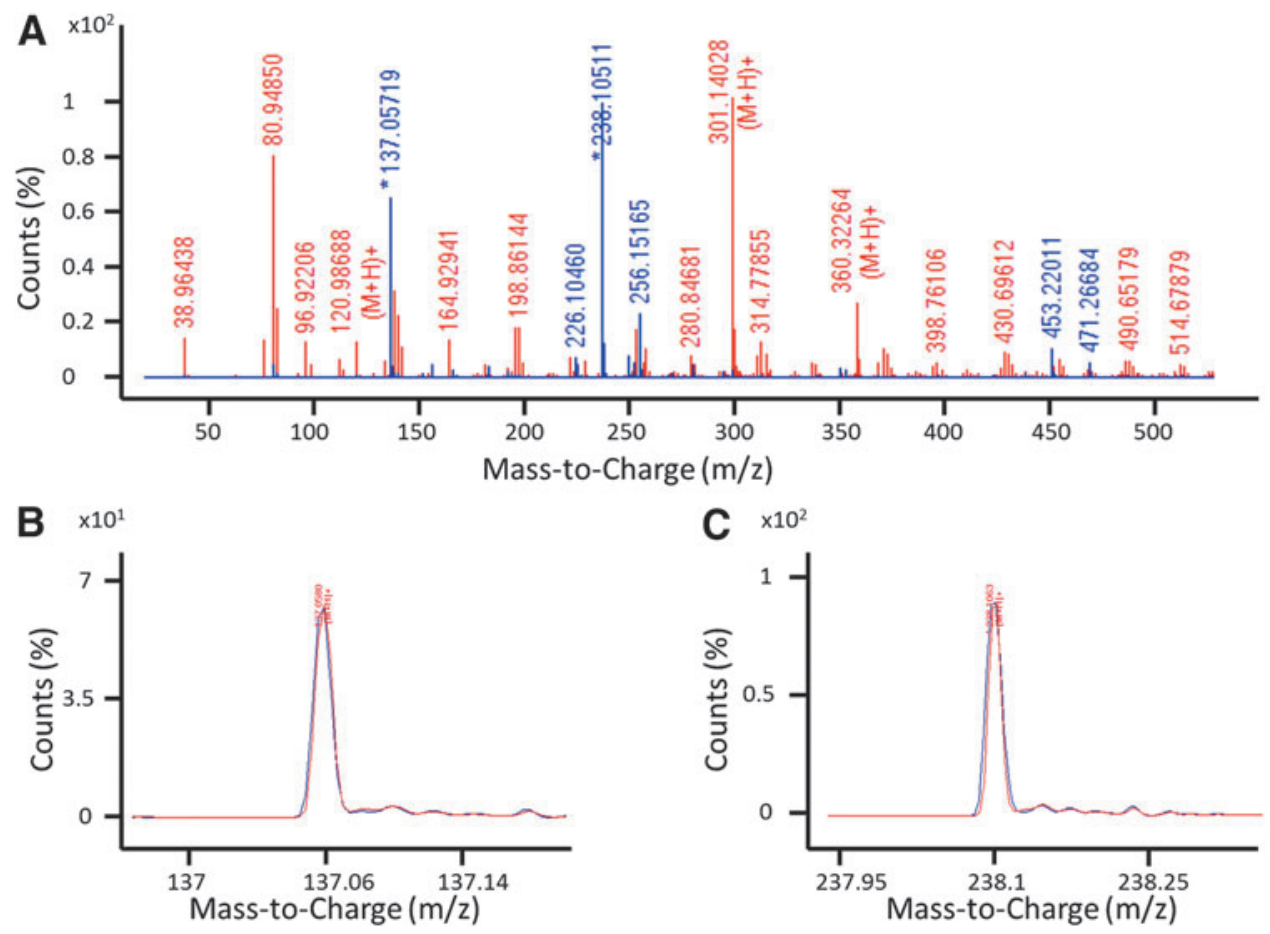

FIG. 3. ESI-TOF data of $1 \times$ PBS incubated in FormLab Flexible tubes. (A) Spectrum of PBS extract Form Flex tube (blue) overlaid with the spectrum of PBS extract from polystyrene tube ( red), (B) overlay of sample 137.0572 peak to ethyl methacrylate standard, and (C) overlay of sample 238.1051 peak to diethyl dimethylaminomethylene malonate standard. PBS, phosphate-buffered saline.

the control: "MED610," "VeroClear," and "Frosted Acrylic." These materials also have similar $\mathrm{OD}_{600}$ values after $24 \mathrm{~h}, 4.345,3.922$, and 3.900, respectively, compared to the polystyrene control tubes, 4.656. The second group of tubes shows growth inhibition: "ClearV2," "Flexible," and "Tango Plus," all with slower initial growth rates and $24 \mathrm{~h} \mathrm{OD} 600$ much lower compared with the control $(1.198,0.590$, and 0.235 , respectively). It was noted, however, that a lawn of colonies could be recovered from plating $100 \mu \mathrm{L}$ of any culture on $\mathrm{LB}+\mathrm{Kan}$ and incubating overnight at $37^{\circ} \mathrm{C}$. This result strongly suggests growth inhibition rather than a bacteriocidal property.

We used ESI-TOF mass spectrometry to investigate whether residual chemicals had leached into the media from the growth-inhibiting FormLabs "Flexible" tubes, as the unpolymerized resin was easily attainable. To best identify additional $\mathrm{m} / \mathrm{z}$ peaks from the printed material and to limit the total number of peaks in the spectra, phosphate-buffered saline (PBS, Cat. no.: D8537; Sigma-Aldrich) was used instead of standard growth media. A $5 \mathrm{~mL}$ aliquot of PBS was placed in FormLabs "Flexible" and standard polystyrene tubes and shaken overnight at $37^{\circ} \mathrm{C}$. Mass spectra of the resulting solutions are shown in Figure 3A. There are clear peaks around 137 and $238 \mathrm{~m} / \mathrm{z}$ in the FormLabs "Flexible" sample that is not observed in the control. These were hypothesized to correspond to ethyl methacrylate and diethyldimethylaminomethylene malonate, respectively, based on predicted chemical composition and information provided in the Material Safety Data Sheet. Standards of these chemicals were acquired for a comparison of the two peaks, which are seen in Figure 3B and C. A standard curve of each chemical was constructed and used to estimate concentrations of $15 \mathrm{mg} /$
$\mathrm{mL}$ ethyl methacrylate and $0.14 \mathrm{mg} / \mathrm{mL}$ diethyldimethylaminomethylene malonate in the PBS sample.

\section{Discussion}

We tested whether these two chemicals were sufficient to inhibit bacterial growth at the concentrations interpreted from the mass spectra. These compounds were included in the media for cultures grown in standard polystyrene tubes, both individually and in combination (Fig. 4). The addition of

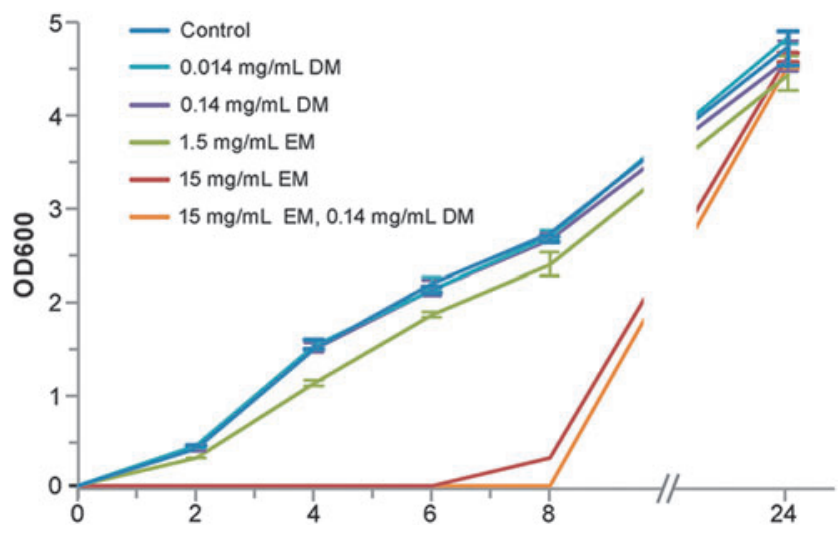

FIG. 4. Growth data from KEIO collection strain JW07403 in polystyrene control tubes in LB media chemically spiked samples with the potential growth inhibitors. Twentyfour hour time course of growth; Error bars represent \pm 1 SD. EM, ethyl methacrylate; DM, dimethylaminomethylene malonate. 


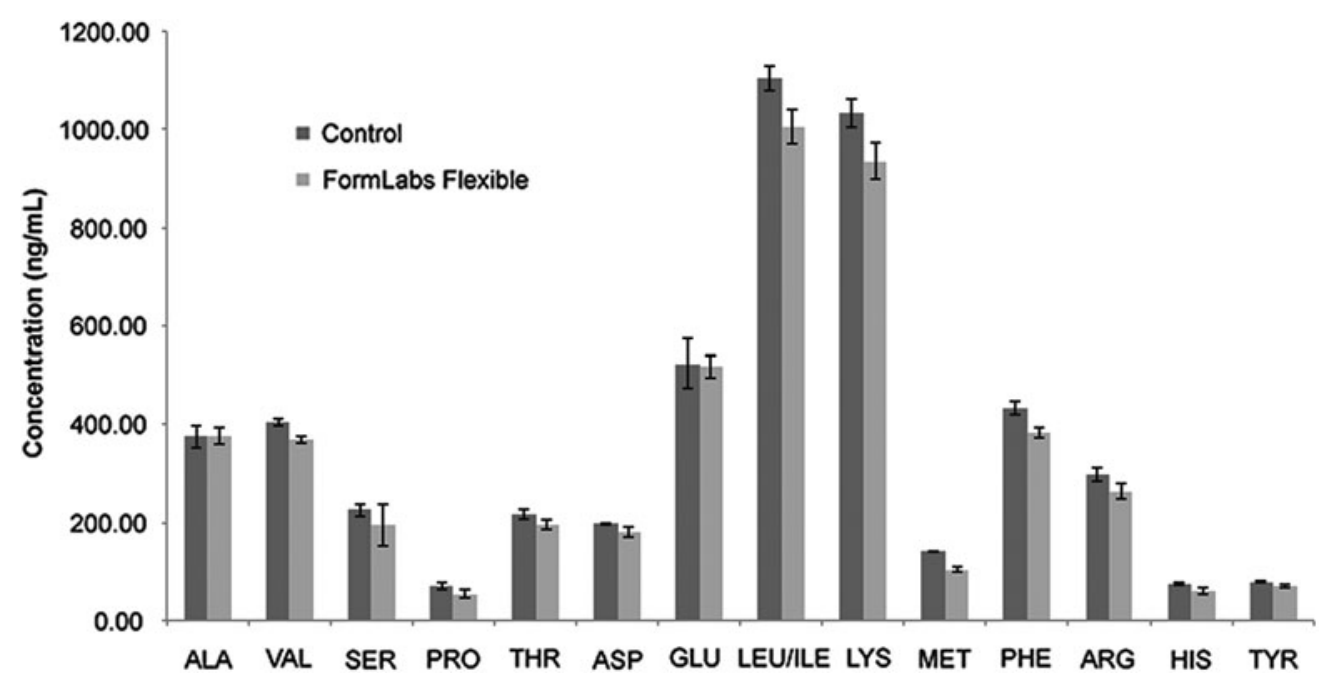

FIG. 5. Mass spectrometry analysis of various amino acids from LB growth media. Incubation of samples occurred overnight at $37^{\circ} \mathrm{C}$ with shaking. Media was in polystyrene control tubes and in FormLabs "Flexible" printed tubes. Note: leucine and isoleucine isomers are indistinguishable under the given method and, thus, reported together. Error bars represent \pm 1 SD. FormLabs "Flexible" results are reported as the average of three tubes.

diethyldimethylaminomethylene malonate did not inhibit growth at concentrations up to $0.14 \mathrm{mg} / \mathrm{mL}$. However, the addition of $15 \mathrm{mg} / \mathrm{mL}$ ethyl methacrylate retarded the course of growth over the first $8 \mathrm{~h}$. At one-tenth of that concentration, growth was only slightly retarded relative to the control. The combination of both the ethyl methacrylate and the dimethylaminomethylene malonate inhibits growth over the first $8 \mathrm{~h}$ with no growth observed even at the 8-h mark, suggesting an additive effect of the two chemicals. However, in all cultures, an optical density was observed after $24 \mathrm{~h}$ that was similar to that of the control tube. As these chemicals were derived from a UV-polymerizable mixture, we suspect that the added chemicals polymerize over the course of the incubation, leading to a decreased concentration of the inhibitory species. We noted that polymerized material had accumulated on the side of the tubes containing $15 \mathrm{mg} / \mathrm{mL}$ ethyl methacrylate. We conclude that potential leaching of unpolymerized chemicals from a printed material should be taken into account when planning a given biological application.

Another hypothesis for growth inhibition is that beneficial components of the growth media (such as amino acids) are being absorbed by the FormLabs "Flexible" resin. To investigate this question, LB broth was incubated in both polystyrene control and FormLabs "Flexible" tubes for $24 \mathrm{~h}$ at $37^{\circ} \mathrm{C}$ with shaking. The samples were then analyzed by mass spectrometry and the concentration in the media of a selection of amino acids was determined by comparison to a commercial amino acid standard. The data, which are found in Figure 5, suggest that there is no depletion of any measured amino acid to an extent that would strongly inhibit growth.

\section{Conclusion}

We have demonstrated an approach for evaluating the potential of ten 3D-printable materials for microbial liquid culture. Using this template, we identified six 3D-printable materials that produced tubes suitable for working with aqueous liquids. The other four materials displayed chal- lenges with physical integrity (e.g., allowing evaporation or releasing fine particles into the growth media). Of the six suitable materials, only three ("MED610," "VeroClear," and "Frosted Acrylic") showed no appreciable growth inhibition of E. coli compared to standard commercial polystyrene tubes. The FormLabs, "Flexibile" and "ClearV2," and the Stratasys "TangoPlus" materials inhibited growth to varying degrees. We were able to further investigate and identify bacteriostatic chemicals that leached into the growth media from a material that displayed growth inhibition (FormLabs "Flexible"). Throughout this process, our goal was not to supplant standard commercial culture tubes (which are generally more economical) with their 3D-printed equivalents. Rather, by reproducing the physical shape and dimensions of a typical culture tube, we sought to provide the best comparison of materials. Understanding which 3Dprintable materials are suitable for microbial growth is an important step in our laboratory for ongoing projects that rapidly prototype new microbial culture environments. We seek to explore and realize the potential of 3D-printable materials in novel devices designed for the study of microbiology and molecular biology and to enable others in doing likewise.

\section{Acknowledgments}

This work is sponsored by the Assistant Secretary of Defense for Research \& Engineering under Air Force Contract No. FA8721-05-C-0002. Opinions, interpretations, conclusions, and recommendations are those of the authors and are not necessarily endorsed by the U.S. Government. We thank Dr. Todd Thorsen and Dr. Rafmag Cabrera at MIT Lincoln Laboratory for support in tube design and thoughtful discussion. The Massachusetts Institute of Technology Lincoln Laboratory Technology Office Innovation Laboratory was used to print tubes using the Connex 350 and FormLabs Form 1+.

\section{Author Disclosure Statement}

No competing financial interests exist. 


\section{References}

1. Berman B. 3-D printing: The new industrial revolution. Bus Horiz 2012;55:155-162.

2. McCullough EJ, Yadavalli VK. Surface modification of fused deposition modeling ABS to enable rapid prototyping of biomedical microdevices. J Mater Process Technol 2013; 213:947-954.

3. Ahn SH, Montero M, Odell D, et al. Anisotropic material properties of fused deposition modeling ABS. Rapid Prototyping J 2002;8:248-257.

4. Rambo CR, Travitzky N, Zimmermann K, Greil P. Synthesis of $\mathrm{TiC} / \mathrm{Ti}-\mathrm{Cu}$ composites by pressureless reactive infiltration of $\mathrm{TiCu}$ alloy into carbon preforms fabricated by 3D-printing. Mater Lett 2005;59:1028-1031.

5. Bergmann C, Lindner M, Zhang W, et al. 3D-printing of bone substitute implants using calcium phosphate and bioactive glasses. J Eur Ceram Soc 2010;30:2563-2567.

6. Marchelli G, Prabhakar R, Storti D, Ganter M. Highly Porous 3D Printed Stoneware Ceramic. Rapid Prototyping J 2011;17:187-194.

7. Xu N, Wei F, Liu X, et al. Reconstruction of the Upper Cervical Spine Using a Personalized 3D-Printed Vertebral Body in an Adolescent With Ewing Sarcoma. Spine. 2016;41:E50-E54.

8. Zhu F, Skommer J, Macdonald NP, et al. Three-dimensional printed millifluidic devices for zebrafish embryo tests. Biomicrofluidics 2015;9:046502.
9. Lücking TH, Sambale F, Schnaars B, et al. 3D-printed individual labware in biosciences by rapid prototyping: In vitro biocompatibility and applications for eukaryotic cell cultures. Eng Life Sci 2015;15:57-64.

10. Baba T, Ara T, Hasegawa M, et al. Construction of Escherichia coli K-12 in-frame, single-gene knockout mutants: the Keio collection. Mol Syst Biol 2006;2: $1-11$.

Address correspondence to: Peter A. Carr

Massachusetts Institute of Technology Lincoln Laboratory 244 Wood Street Lexington, MA 02420

E-mail: carr@1l.mit.edu

and

David S. Kong

Massachusetts Institute of Technology Lincoln Laboratory 244 Wood Street Lexington, MA 02420

E-mail: dkong@11.mit.edu 